\title{
THE SURGICAL TREATMENT OF SUPRAVALVULAR STENOSIS BASED ON TWO CASES
}

\author{
BY \\ SAM NORDSTRÖM AND TORSTEN SILANDER \\ From the Clinic for Thoracic Surgery, Karolinska Sjukhuset, Stockholm, Sweden
}

(RECEIVED FOR PUBLICATION JANUARY 17, 1962)

An increasing number of cases of supravalvular aortic stenosis have been reported in recent years. In those hitherto described, the stricture has consisted of either a fibrous membrane directly adjacent to the cusps or a true stenosis at the level of the upper border of the sinus of Valsalva where, as mentioned by Kreel, Reiss, Strauss, Blumenthal, and Baronofsky (1959), a slight, circular inward bulge in the aortic wall is normally present. Formerly, attempts at operative treatment of supravalvular aortic stenosis met with failure. However, in 1961, McGoon, Mankin, Vlad, and Kirklin reported three cases in which operation had been performed at the Mayo Clinic with excellent results. In these the supravalvular stenosis had been split and a patch graft sutured into the defect. Starr, Dotter, and Griswold (1961) also reported a patient successfully operated on by the same technique. During the past two years two patients who had supravalvular aortic stenosis have successfully undergone operation at the Thoracic Clinic, Karolinska Sjukhuset; an account of them seems to be warranted.

\section{CASE Reports}

CASE 1.-A 28-year-old woman had had organic heart disease diagnosed in childhood. Her physical activity was slightly limited. A high-frequency, early to mid-systolic murmur was audible, with a maximum over the second right interspace. The heart volume was $430 \mathrm{ml} . / \mathrm{m}^{2}$ of body surface. The E.C.G. showed left ventricular hypertrophy. At cardiac catheterization the pressure was $233 / 19 \mathrm{~mm}$. $\mathrm{Hg}$ in the left ventricle and $111 / 61 \mathrm{~mm}$. $\mathrm{Hg}$ in the aorta (i.e., indicative of tight aortic stenosis). In addition, there were signs of moderate pulmonary hypertension with raised pulmonary resistance. No angiocardiographic examination was made and the condition was diagnosed as congenital aortic stenosis.

Operation was performed on September 16, 1958, under extracorporeal circulation with the heart-lung machine of Crafoord and Senning. A few centimetres above the aortic valve the wall of the ascending aorta was abnormal; the vessel was partly occluded by a thickening, of which the main part protruded into the lumen of the aorta. As much as possible of this obstruction was removed, making an opening ㅇ in the lumen of the aorta slightly wider than an index finger. The aortic cusps were normal. The $\vec{c}$ aortic wall was then sutured. Post-operatively, a systolic murmur of the same type and localization as $\overparen{D}$ was heard before operation was present, but it was $\vec{\bullet}$ fainter, of lower frequency, and less harsh. The o patient was discharged from hospital in good condition just over three weeks after operation.

She was readmitted to hospital for follow-up examination in September, 1961. She had been asymptomatic since operation, and worked as an assistant hospital nurse on night duty. In 1960 she $\mathbb{D}$ had given birth to a child. Both pregnancy and delivery were uncomplicated. The heart volume was $400 \mathrm{ml}$. $/ \mathrm{m}^{2}$ of body surface compared with $430 \mathrm{ml}$./ $\mathrm{m}^{2}$ pre-operatively. The E.C.G. no longer showed hypertrophy of the left ventricle, and depression of the $S-T$ intervals and $T$ waves was less. At phonocardiography a medium-frequency, diamond-shaped pansystolic murmur was recorded, with a maximum over the second right interspace, but of lower intensity than previously. Angiocardiography showed that a stricture with an inner diameter of $1.5 \mathrm{~cm}$. was still present at the site of the supravalvular stenosis (Fig. 1). The systolic pressure gradient across the stenosis was, however, only $37 \mathrm{~mm}$. Hg compared with $122 \mathrm{~mm}$. $\mathrm{Hg}$ pre-operatively (Table I). Consequently, we felt that there was no indication for further intervention.

TABLE I

PRE- AND POST-OPERATIVE CARDIAC CATHETERIZATION IN TWO CASES OF SUPRAVALVULAR AORTIC STENOSIS

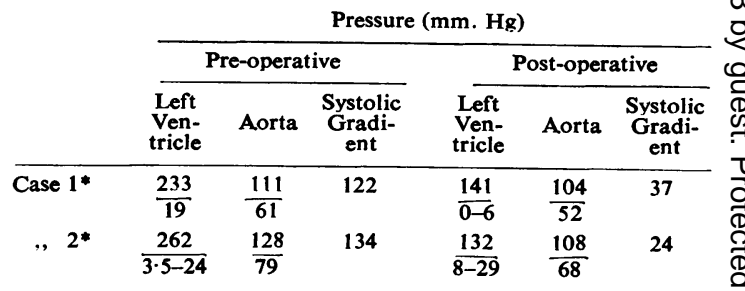

* In Case 1, operation consisted of local excision of the stenosis; and in Case 2, of splitting the aorta through the stenosis and insertion of a patch graft. 


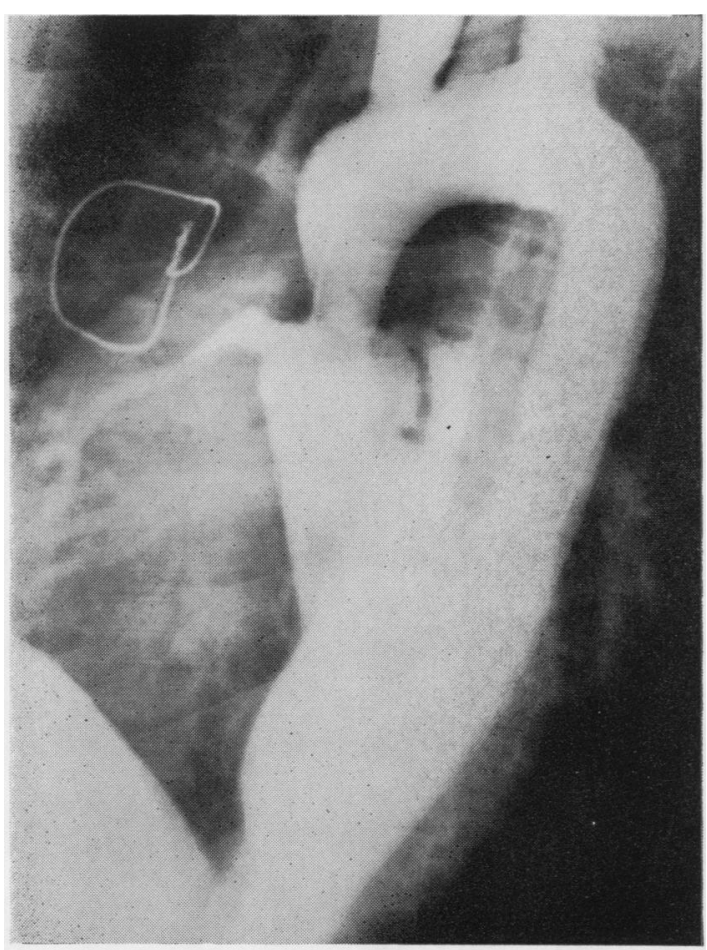

FIG. $1 a$

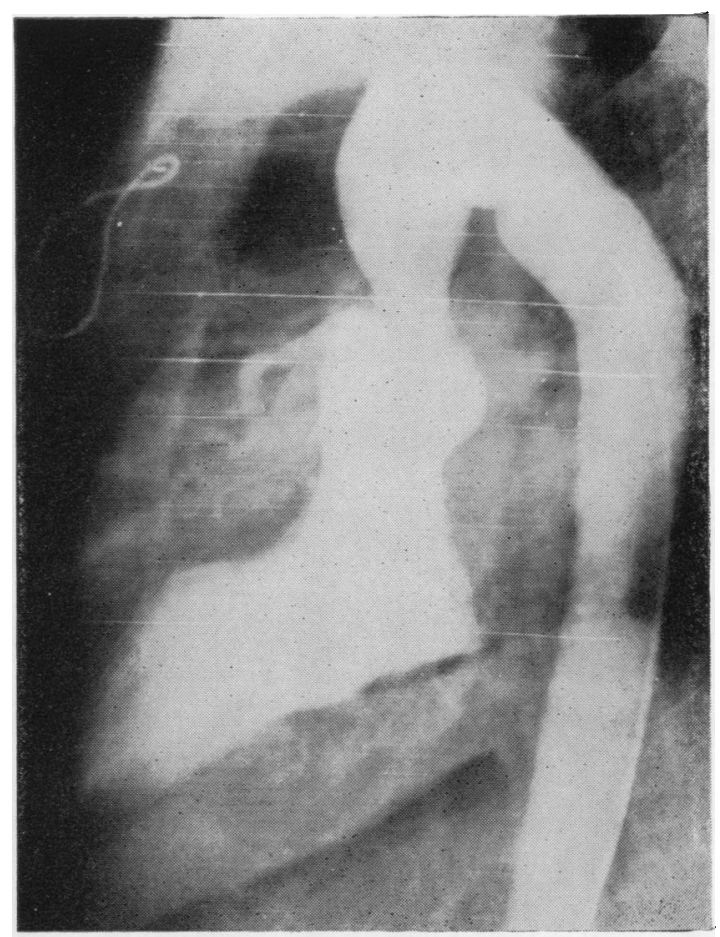

FIG. $1 b$

Fig. $1(a)$ and $(b)$.-Case 1. Post-operative angiocardiography (Dr. Eric Carlsson, Thoracic Clinic) in two planes in a case of supravalvular aortic stenosis. Operation consisted of local excision of the stenosis. Post-operative pressure gradient across the former siteof stenosis $37 \mathrm{~mm}$. $\mathbf{H g}$.

CASE 2.-An 8-year-old boy had congenital organic heart disease but no appreciable functional limitation. At the age of 7 he had had a subarachnoid haemorrhage. Investigation at another hospital in 1959 disclosed a systolic thrill over the whole praecordium transmitted to the vessels of the neck, as well as a high-frequency systolic murmur (grade 6) with a maximum over the second right interspace. Although the peripheral pulsations were faint, the blood pressure could be measured in both the arms and legs $(100 /$ $75 \mathrm{~mm}$. $\mathrm{Hg}$ ). The heart volume was $620 \mathrm{ml} . / \mathrm{m}^{2}$ of body surface. Moderate stasis was present in the pulmonary circulation. At cardiac catheterization the pressure was $262 / 3.5-24 \mathrm{~mm}$. $\mathrm{Hg}$ in the left ventricle and $128 / 79 \mathrm{~mm}$. $\mathrm{Hg}$ in the aorta. Angiocardiography showed marked hypertrophy of the left ventricle as well as stenosis of the ascending aorta for slightly over $1.5 \mathrm{~cm}$., starting a few centimetres above the plane of the aortic valve. At the narrowest site the lumen was $6 \mathrm{~mm}$. in diameter (Fig. 2). Among the findings at cerebral angiography was an aneurysm of the posterior cerebral artery. It was decided to operate on the aortic stenosis in the first place, this being the more vital indication.

Operation was performed on June 14, 1959, under extracorporeal circulation with the heart-lung machine of Crafoord and Senning. The ascending aorta was found to be greatly stenosed a few centimetres above the valvular plane. An anterior incisiont was made in the vessel, extending from above the coarctation to below it. At the narrowest site the diameter of the lumen was $6 \mathrm{~mm}$. The aortic cusps were normal. A diamond-shaped dacron graft, $3.5 \mathrm{~cm}$. long and $2.5 \mathrm{~cm}$. wide, was sutured into the incision in the aorta. After an uneventful post-operative course the patient was sent on the nineteenth day to another hospital for further investigation of the cerebral vascular anomalies.

He was readmitted for follow-up examination in August, 1961, two years after operation. No operation had been performed for the cerebral vascular anomalies since it was considered to be too risky. The boy's physical activity was normal. His heart volume was $400 \mathrm{ml} . / \mathrm{m}^{2}$ of body surface as compared to $620 \mathrm{ml} . / \mathrm{m}^{2}$ pre-operatively. A faint (grade 3 ) systolic murmur was audible with a maximum over the second right interspace. Angiocardiography, with injection of contrast medium into the left ventricle. showed that there was no longer any impediment to passage in the ascending aorta. An indentation about $3 \mathrm{~mm}$. deep was visible, corresponding to the site of the graft (Fig. 3). The pressure conditions at cardiac catheterization are seen in Table $I$. 


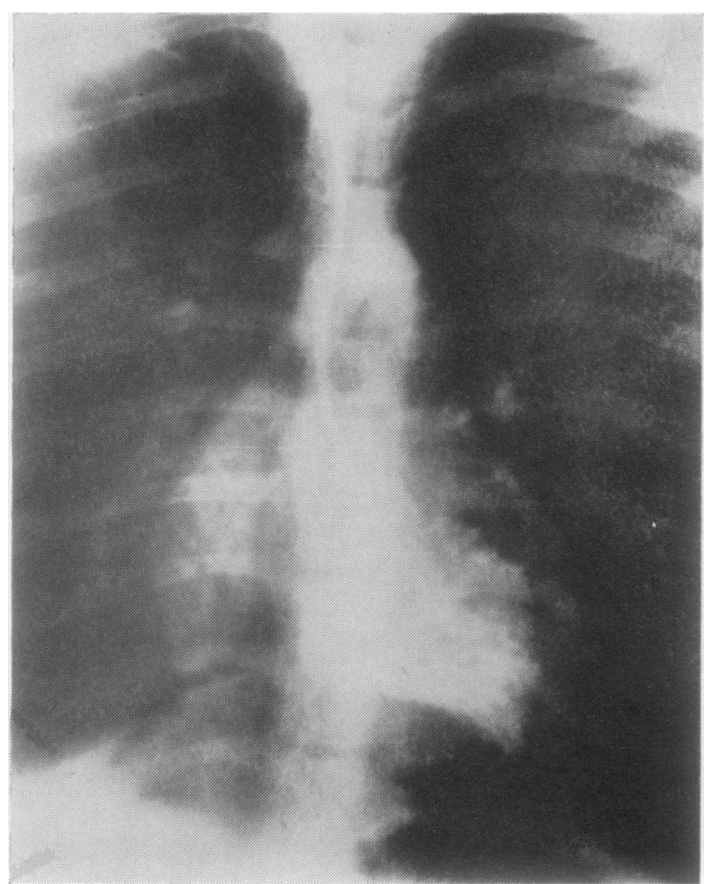

FIG, $2 a$

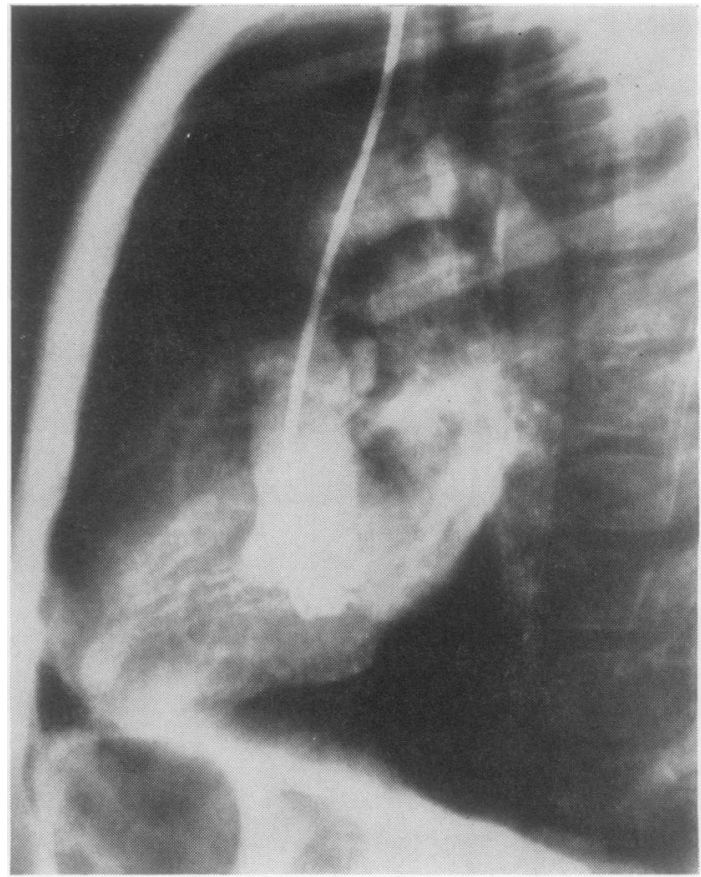

FIG. $2 b$

Fig. $2(a)$ and $(b)$.-Case 2. Pre-operative angiocardiography in two planes in a case of supravalvular aortic stenosis. Pre-operative pressure gradient across the stenosis $134 \mathrm{~mm}$. Hg. (Courtesy of D $i$. G. Jönsson, Södersjukhuset, Stockholm.)

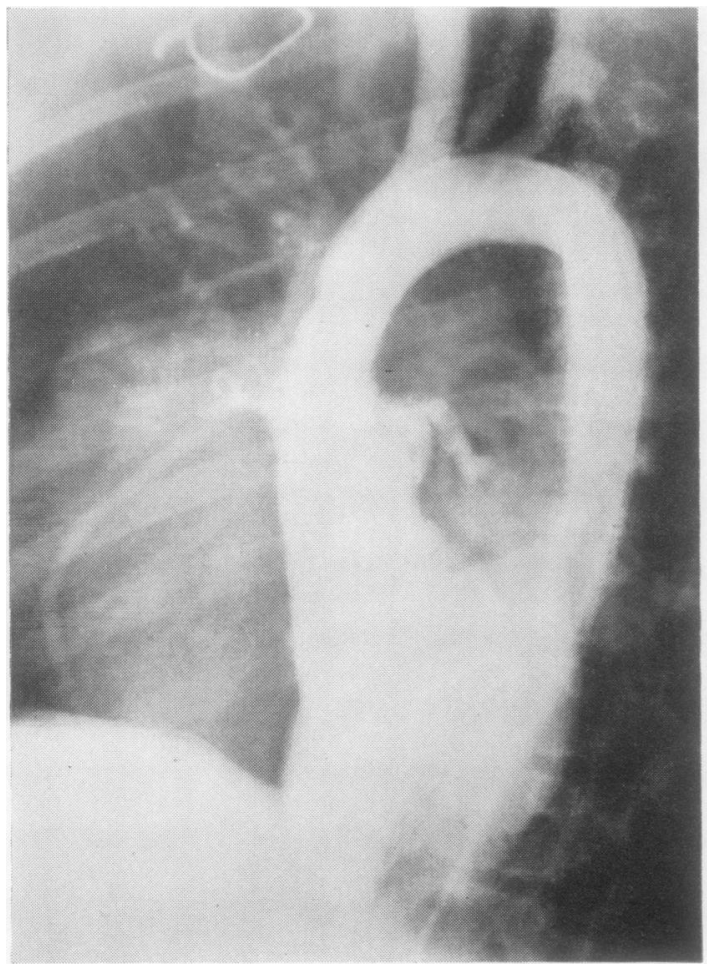

FIG, $3 a$

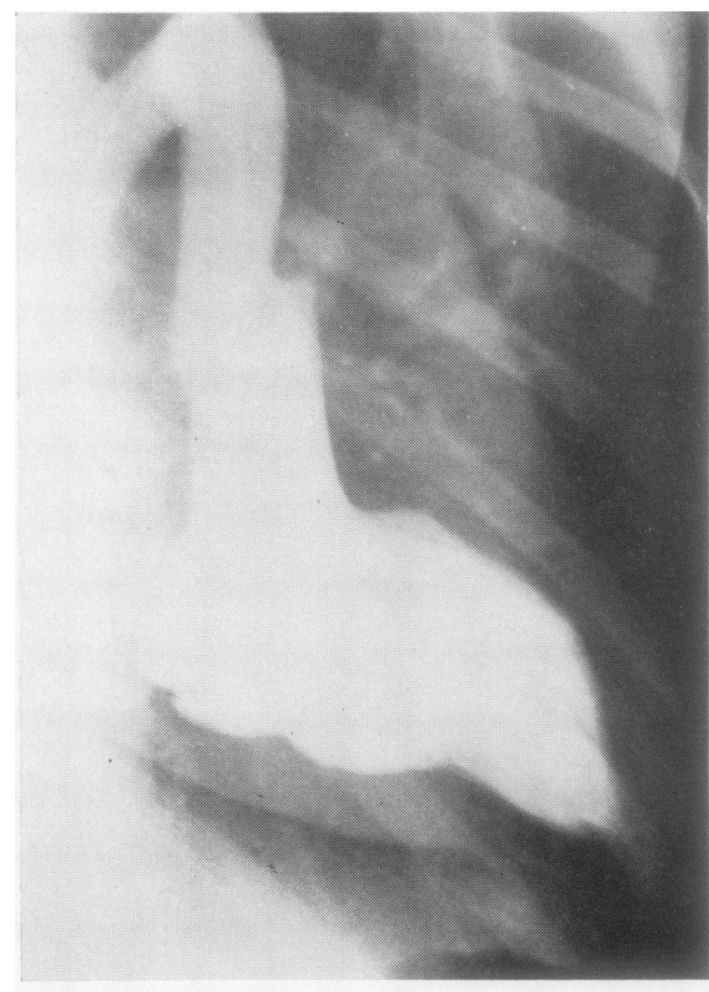

FIG. $3 b$

FIG. $3(a)$ and $(b)$.-Case 2. Post-operative angiocardiography (Dr. Eric Carlsson, Thoracic Clinic). Operation consisted of splitting the arta through the stenosis and insertion of a patch graft. Post-operative pressure gradient $24 \mathrm{~mm}$. $\mathrm{Hg}$. 


\section{Discussion}

In both our cases the stenosis was at the typical site, i.e., at the upper border of the sinus of Valsalva. A remarkable feature in Case 2 is that the stricture involved a segment of the aorta more than $1.5 \mathrm{~cm}$. long. Both radiologically and macroscopically it resembled a coarctation of the aorta. No biopsy was done. In Case 1 the stenosis was removed and examined microscopically. It was found to contain smooth muscle, indicating that inward bulging of the media was responsible for the stenosis. According to Edwards (1953) this medial deformity is the anatomical basis of constriction of the lumen in true coarctation of the aorta.

In 1959 Eiken described a case of coarctation of the aorta central to the origin of the innominate artery. He stated that he had been unable to trace any case described earlier in the literature. Eiken's patient was a 35-year-old woman with such pronounced calcification of the aortic wall that the coarctation was directly visible on plain radiographs and was easily depicted by tomography. In view of the advanced calcifications her condition was regarded as inoperable. Consequently no microscopical diagnosis was available.

At physical examination these cases present the same features as cases of valvular aortic stenosis. The diagnosis is established by the withdrawal curve on catheterization from either the aortic side or the left side of the heart. The most certain way of obtaining a diagnosis is by angiocardiography or aortography. In our Case 1 pre-operative angiography had not been performed. The condition was diagnosed as tight aortic stenosis, on the basis of the physical findings and the results of catheterization. At operation we were surprised to find completely normal aortic cusps and an aorta of ordinary width in the valvular plane. We found instead a stenosis of the diaphragm type with thickening of the aortic wall above the valve. In Case 2 the anatomical conditions had been depicted at pre-operative angiocardiography, and we could therefore make a direct attack on the malformation at operation. In the case reported by Kreel et al. there was not only supravalvular aortic stenosis but also valvular lesions the nature of which could not be identified at operation and which led to the patient's death.

With the technique now used for angiocardiography, this examination is associated with a relatively small risk for the patient. Consequently, in our opinion, all patients with physical signs of valvular aortic stenosis should undergo an angiocardiographic examination. This is partly to avoid unexpected coincident anomalies and partly, but not least, to obtain an exact pre-operative diagnosis. We, in contrast to McGoon et al., do not believe that, because planning of the operation is the same in the presence of subvalvular, valvular, and supravalvular aortic stenosis, this is sufficient reason to abstain from pre-operative angiocardiography.

Surgical Technique.-The operation necessitates use of the heart-lung machine. The most suitable surgical procedure seems to us to be that applied in our Case 2, which is essentially the same as that described by Starr et al. and by McGoon et al. After a midline incision and cleaving of the sternum, the venae cavae and one of the femoral arteries are cannulated. Continuous deloading of the left side of the heart is procured by a cannula in the left atrium. When perfusion has been started, the aorta is occluded and an arched incision is made in its anterior wall through the stricture and well down into the noncoronary aortic sinus. A diamond-shaped dacron or teflon graft of appropriate size is then sutured into the defect. The technique is analogous to that described by Senning (1959), among others. The graft should be large enough to cover the whole incision, with its widest part at the level of the most stenosed segment. An adventitial flap, prepared in advance, can be swept over the graft and suture lines.

It is important for the suction cannula in the left atrium to be shut off before suturing of the graft is finished. This gives the left side of the heart time to become filled with blood, with expulsion of all air that may have been sucked into the left atrium. Use of the left atrial cannula provides an operative field that is as bloodless as possible. Consequently, the ordinary coronary sucker tip, which masks the operative field, need be used only to a limited extent, as Starr et al. have also pointed out. Separate coronary perfusion seems to be superfluous as occlusion scarcely requires to exceed 15 to 20 minutes.

The surgical technique used in our Case 1 does not appear to have been described earlier. When the follow-up examination was made, we were prepared to reoperate on the patient, with insertion of a graft. Since she was, however, asymptomatic and had a decreased heart volume and a systolic pressure gradient of only $37 \mathrm{~mm}$. $\mathrm{Hg}$, we regarded the results as highly satisfactory. 


\section{SUMMARY}

An account is given of two patients with supravalvular aortic stenosis, in whom operation was successfully performed by different methods. The surgical technique is discussed, and widening of the stenosed supravalvular segment with a patch graft is recommended. The importance is stressed of exact pre-operative visualization of the anatomical conditions by angiocardiography. The excellent results of operation could be verified in both cases by follow-up examination three and two years later, respectively.

\section{REFERENCES}

Edwards, J. E. (1953). Amer. J. clin. Path., 23, 1240.

Eiken, M. (1959). Acta med. scand., 165, 235.

Kreel, I., Reiss, R., Strauss, L., Blumenthal, S., and Baronofsky, I. D. (1959). Ann. Surg., 149, 519.

McGoon, D. C., Mankin, H. T., Vlad, P., and Kirklin, J. W. (1961) J. thorac. Surg., 41, 125.

Senning, A. (1959), Acta chir. scand., 118, 81.

Starr. A., Dotter, C., and Griswold, H. (1961). J. thorac. Surg., 41, i34. 